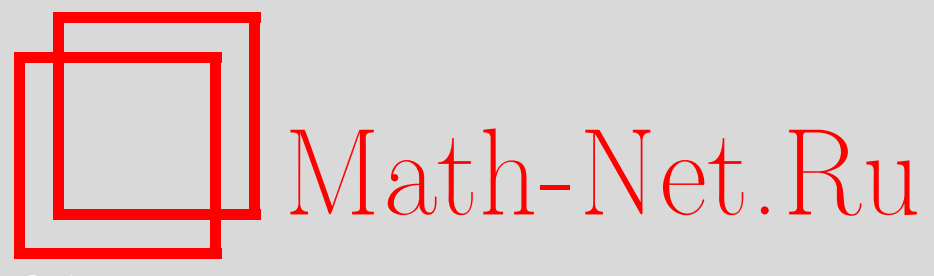

А. Л. Бекларян, О существовании решений первой краевой задачи для эллиптических систем высокого порядка в неограниченных областях, Матем. заметки, 2014, том 96, выпуск 2, 310-313

DOI: https://doi.org/10.4213/mzm10485

Использование Общероссийского математического портала Math-Net.Ru подразумевает, что вы прочитали и согласны с пользовательским соглашением http://www.mathnet.ru/rus/agreement

Параметры загрузки:

IP : 35.173 .219 .149

26 апреля 2023 г., 13:30:05

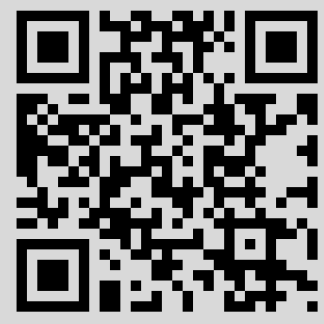




\section{О существовании решений первой краевой задачи для эллиптических систем высокого порядка в неограниченных областях}

\section{А. Л. Бекларян}

1. Введение. Пусть $\Omega$ - неограниченное открытое подмножество $\mathbb{R}^{n}, n \geqslant 2$. Обозначим через $B_{\rho}^{x}$ - открытый шар в $\mathbb{R}^{n}$ радиуса $\rho>0$ с центром в точке $x$. Если $x=0$, пишем $B_{\rho}$ вместо $B_{\rho}^{x}$. Как это принято, под $W_{2, \text { loc }}^{m}(\Omega)$ будем подразумевать множество функций из $\mathscr{D}^{\prime}(\Omega)$, принадлежащих пространствам $W_{2}^{m}\left(\Omega \cap B_{\rho}\right)$ для всех вещественных чисел $\rho>0$ [1]. При этом через $\stackrel{\circ}{W}_{2, \text { loc }}^{m}(\Omega)$ будем обозначать подмножество пространства $W_{2, \text { loc }}^{m}\left(\mathbb{R}^{n}\right)$, которое получается замыканием $C_{0}^{\infty}(\Omega)$ в системе полунорм $\|u\|_{W_{2}^{m}\left(\Omega \cap B_{\rho}\right)}, \rho>0$. Далее, следуя [2; $\S 1.1]$, под $L_{2}^{m}(\Omega)$ будем подразумевать пространство обобщенных функций, все производные порядка $m$ которых принадлежат $L_{2}(\Omega)$; другими словами,

$$
L_{2}^{m}(\Omega)=\left\{f \in \mathscr{D}^{\prime}(\Omega): \int_{\Omega} \sum_{|\alpha|=m}\left|\partial^{\alpha} f\right|^{2} d x<\infty\right\},
$$

где $\alpha=\left(\alpha_{1}, \ldots, \alpha_{n}\right)$ - мультииндекс, $|\alpha|=\alpha_{1}+\cdots+\alpha_{n}$.

Пусть $\omega \subset \mathbb{R}^{n}$ - открытое множество, $\mathscr{K} \subset \omega-$ компакт, $\mathfrak{P}_{m-1}-$ пространство многочленов вида

$$
p(x)=\sum c_{\alpha} x^{\alpha}, \quad c_{\alpha} \in \mathbb{R}, \quad|\alpha| \leqslant m-1 .
$$

Обозначим через $\Phi_{\varphi}(\mathscr{K}, \omega), \varphi \in W_{2, \text { loc }}^{m}\left(\mathbb{R}^{n}\right)$, множество функций $\psi \in C_{0}^{\infty}(\omega)$, удовлетворяющих условию $\psi=\varphi$ в окрестности $\mathscr{K}$, или, другими словами, $\psi-\varphi \in \stackrel{\circ}{W}_{2, \text { loc }}^{m}\left(\mathbb{R}^{n} \backslash \mathscr{K}\right)$. Положим

$$
\Psi(\mathscr{K}, \omega)=\left\{\psi \in C_{0}^{\infty}(\omega): \psi=1 \text { в окрестности } \mathscr{K}\right\} .
$$

Емкостъю компакта $\mathscr{K}$ относительно открытого множества $\omega$ назовем величину

$$
\operatorname{cap}_{\varphi}(\mathscr{K}, \omega)=\inf _{\psi \in \Phi_{\varphi}(\mathscr{K}, \omega)} \int_{\omega} \sum_{|\alpha|=m}\left|\partial^{\alpha} \psi\right|^{2} d x .
$$

Емкость произвольного замкнутого в $\mathbb{R}^{n}$ множества $E \subset \omega$ определяется формулой

$$
\operatorname{cap}_{\varphi}(E, \omega)=\sup _{\mathscr{K}} \operatorname{cap}_{\varphi}(\mathscr{K}, \omega),
$$

где точная верхняя грань в правой части берется по всем компактам $\mathscr{K} \subset E$. Если $\omega=\mathbb{R}^{n}$, то вместо сар $\varphi\left(E, \mathbb{R}^{n}\right)$ будем писать сар $\varphi(E)$.

Нам также потребуется следующая емкость $[2 ; \S 9.1]$ :

$$
\operatorname{Cap}\left(\mathscr{K}, W_{2}^{m}(\omega)\right)=\inf _{\psi \in \Psi(\mathscr{K}, \omega)}\left(\int_{\omega} \sum_{|\alpha|=m}\left|\partial^{\alpha} \psi\right|^{2} d x+\int_{\omega}|\psi|^{2} d x\right) .
$$

Как и выше, емкость произвольного замкнутого в $\mathbb{R}^{n}$ множества $E \subset \omega$ определяется формулой

$$
\operatorname{Cap}\left(E, W_{2}^{m}(\omega)\right)=\sup _{\mathscr{K}} \operatorname{Cap}\left(\mathscr{K}, W_{2}^{m}(\omega)\right),
$$

где точную верхнюю грань в правой части следует брать по всем компактам $\mathscr{K} \subset E$.

Вопросы, рассматриваемые в настоящей заметке, ранее исследовались в работах [3]-[5].

DOI: $10.4213 / \mathrm{mzm} 10485$

(C) А. Л. БЕкЛАРян, 2014 
2. Постановка задачи. Здесь и далее $L$ - дивергентный оператор вида

$$
L=\sum_{|\alpha|=|\beta|=m} \partial^{\alpha}\left(a_{\alpha \beta}(x) \partial^{\beta}\right)
$$

где $a_{\alpha \beta}(x)$ - измеримые и ограниченные функции аргумента $x \in \mathbb{R}^{n}$ со значениями в пространстве $(N \times N)$-матриц. Потребуем, чтобы для всякой бесконечно гладкой функции $u: \mathbb{R}^{n} \rightarrow \mathbb{R}^{N}$ с компактным носителем имело место неравенство

$$
\int \sum_{|\alpha|=|\beta|=m} a_{\alpha \beta} \partial^{\beta} u \partial^{\alpha} u d x \geqslant \gamma \int \sum_{|\alpha|=m}\left|\partial^{\alpha} u\right|^{2} d x, \quad \gamma>0 .
$$

Здесь и везде ниже мы для краткости обозначаем

$$
a_{\alpha \beta} \partial^{\beta} u \partial^{\alpha} v=\left(a_{\alpha \beta} \partial^{\beta} u, \partial^{\alpha} v\right), \quad\left|\partial^{\alpha} u\right|^{2}=\sum_{i=1}^{N}\left|\partial^{\alpha} u_{i}\right|^{2},
$$

где $(\cdot, \cdot)$ - скалярное произведение в $\mathbb{R}^{N}$.

Решением задачи Дирихле

$$
\begin{cases}L u=0 & \text { в } \Omega, \\ \left.\partial^{\alpha} u\right|_{\partial \Omega}=\partial^{\alpha} \varphi, & |\alpha| \leqslant m-1,\end{cases}
$$

где $\varphi=\left(\varphi_{1}, \ldots, \varphi_{N}\right) \in W_{2, \text { loc }}^{m}\left(\mathbb{R}^{n}\right)$, называется функция $u \in W_{2, \text { loc }}^{m}(\Omega)$, удовлетворяющая следующим условиям:

1) $u-\varphi \in \stackrel{\circ}{W}_{2, \text { lос }}^{m}(\Omega)$, т.е. $(u-\varphi) \eta \in \stackrel{\circ}{W}_{2}^{m}(\Omega)$ для любой функции $\eta \in C_{0}^{\infty}\left(\mathbb{R}^{n}\right)$;

2) функция $u$ имеет ограниченный интеграл Дирихле

$$
\int_{\Omega} \sum_{|\alpha|=m}\left|\partial^{\alpha} u\right|^{2} d x<\infty
$$

3) справедливо интегральное тождество

$$
\int_{\Omega} \sum_{|\alpha|=|\beta|=m} a_{\alpha \beta}(x) \partial^{\beta} u \partial^{\alpha} \psi d x=0
$$

для любой вектор-функции $\psi \in C_{0}^{\infty}(\Omega)$.

\section{3. Основные результаты.}

Teоpema 1. Пусть

$$
\operatorname{cap}_{\varphi_{i}-p_{i}}\left(\mathbb{R}^{n} \backslash \Omega\right)<\infty \quad \text { для некоторых многочленов } p_{i} \in \mathfrak{P}_{m-1}, \quad i=1, \ldots, N .
$$

Тогда задача (1) имеет решение.

Теорема 2. Пусть задача (1) имеет решение и верно, что

$$
\int_{\mathbb{R}^{n} \backslash \Omega} \sum_{|\alpha|=m}\left|\partial^{\alpha} \varphi\right|^{2} d x<\infty .
$$

Тогда существуют многочлены $p_{i} \in \mathfrak{P}_{m-1}$ такие, что

$$
\operatorname{cap}_{\varphi_{i}-p_{i}}\left(\mathbb{R}^{n} \backslash \Omega\right)<\infty, \quad i=1, \ldots, N .
$$


Теорема 3. Для всякой функиии $\psi \in W_{2, \text { lос }}^{m}\left(\mathbb{R}^{n}\right)$ условие

$$
\operatorname{cap}_{\psi}\left(\mathbb{R}^{n} \backslash \Omega\right)<\infty
$$

эквивалентно неравенству

$$
\sum_{k=1}^{\infty} \operatorname{cap}_{\psi}\left(\left(\bar{B}_{r_{k+1}} \backslash B_{r_{k-1}}\right) \cap\left(\mathbb{R}^{n} \backslash \Omega\right), B_{r_{k+2}} \backslash \bar{B}_{r_{k-2}}\right)<\infty,
$$

где

$$
r_{k}= \begin{cases}2^{2^{k}}, & \text { если } n \leqslant 2 m \text { и } п \text { четно } \\ 2^{k} & \text { в остальных случалх }\end{cases}
$$

Пусть $\omega \subset \mathbb{R}^{n}$ - ограниченная область, удовлетворяющая условию конуса $[2 ; \S 1.1 .9]$ и $\mu-$ мера в $\omega$ такая, что

$$
\sup _{x \in \mathbb{R}^{n}, \rho>0} \rho^{-s} \mu\left(B_{\rho}^{x} \cap \omega\right)<\infty,
$$

где $s>0$. Тогда согласно [2; $\S 1.4 .5]$ для любой функции $v \in W_{2}^{m}(\omega)$ существует многочлен $p \in \mathfrak{P}_{m-1}$ такой, что

$$
\sigma_{\beta}(\omega, \mu)\left\|\partial^{\beta}(v-p)\right\|_{L_{2}(\omega, \mu)} \leqslant \sum_{|\alpha|=m}\left\|\partial^{\alpha} v\right\|_{L_{2}(\omega)}
$$

при

$$
m-\frac{n}{2} \leqslant|\beta|<m-\frac{n-s}{2}, \quad s \leqslant n .
$$

Если же выполнено условие

$$
|\beta|<m-\frac{n}{2}
$$

то справедливо неравенство

$$
\sigma_{\beta}(\omega, \mu)\left\|\partial^{\beta}(v-p)\right\|_{C(\omega)} \leqslant \sum_{|\alpha|=m}\left\|\partial^{\alpha} v\right\|_{L_{2}(\omega)},
$$

где постоянная $\sigma_{\beta}(\omega, \mu)>0$ в обоих случаях не зависит от $v$.

Теорема 4. Пусть задача (1) имеет решение, и пусть $\mu_{i}-$ семейство мер в $\omega_{i}$, где $\omega_{i}$, $i=1,2, \ldots$, - попарно не пересекающиеся ограниченные области в $\mathbb{R}^{n}$, удовлетворяющие условию конуса и такие, что

$$
\sup _{x \in \mathbb{R}^{n}, \rho>0} \rho^{s} \mu_{i}\left(B_{\rho}^{x} \cap \omega_{i}\right)<\infty, \quad \sum_{i=1}^{\infty} \int_{\omega_{i} \backslash \Omega} \sum_{|\alpha|=m}\left|\partial^{\alpha} \varphi\right|^{2} d x<\infty .
$$

Пусть также для некоторого мультииндекса $\beta$ выполнено условие (3). Положим

$$
m_{i}(\varphi)=\inf _{p}\left\|\partial^{\beta}(\varphi-p)\right\|_{L_{2}\left(\omega_{i} \backslash \Omega, \mu_{i}\right)},
$$

где точная нижняя грань берется по всем

$$
p \in \underbrace{\mathfrak{P}_{m-1} \times \cdots \times \mathfrak{P}_{m-1}}_{N} .
$$

Тогда

$$
\sum_{i=1}^{\infty} \sigma_{\beta}^{2}\left(\omega_{i}, \mu_{i}\right) m_{i}^{2}(\varphi)<\infty,
$$

где $\sigma\left(\omega_{i}, \mu_{i}\right)$ - коэфбициент в неравенстве (2). 
Теорема 5. Пусть в предположениях теоремы 4 вместо условия (3) выполнено условие (4). Тогда будет справедливо соотношение (6), где

$$
m_{i}(\varphi)=\inf _{p}\left\|\partial^{\beta}(\varphi-p)\right\|_{C\left(\omega_{i} \backslash \Omega\right)},
$$

где точная ниюняя грань берется по всем $p \in \underbrace{\mathfrak{P}_{m-1} \times \cdots \times \mathfrak{P}_{m-1}}_{N}$.

Автор выражает благодарность профессору А. А. Конькову за постановку задачи и внимание, проявленное к автору в процессе ее решения.

\section{СПИСОК ЦИТИРОВАННОЙ ЛИТЕРАТУРЫ}

[1] О. А. Ладыженская, Н. Н. Уральцева, Линейные и квазилинейные уравнения эллиптического типа, Наука, М., 1973. [2] В. Г. Мазья, Пространства С.Л. Соболева, Изд-во Ленинградск. ун-та, Л., 1985. [3] В.А. Кондратьев, Тр. ММО, 16, Изд-во Моск. ун-та, M., 1967, 293-318. [4] А. А. Коньков, Матем. сб., 184:12 (1993), 23-52. [5] В. Г. Мазья, С. В. Поборчий, Проблемы матем. анализа, 53 (2011), 95-112.

\section{А. Л. Бекларян}

Поступило

Московский государственный

университет им. М. В. Ломоносова

E-mail: beklaryan@hotmail.com 